\title{
An investigation of co-speech gesture production during action description in Parkinson's disease
}

\author{
Rebecca A. Cleary ${ }^{\mathrm{a}, \mathrm{e}, \mathrm{f}}$, Ellen Poliakoff ${ }^{\mathrm{b}, *}$, Adam Galpin ${ }^{\mathrm{c}}$, Jeremy P.R. Dick ${ }^{\mathrm{d}}$, Judith Holler ${ }^{\mathrm{b}, \mathrm{g}}$ \\ ${ }^{a}$ The National Hospital for Neurology E' Neurosurgery, Queen Square, London WC1N 3BG, UK \\ ${ }^{\mathrm{b}}$ School of Psychological Sciences, University of Manchester, Manchester M13 9PL, UK \\ ${ }^{\mathrm{c}}$ Directorate of Psychology, Counselling and Psychotherapy, University of Salford, Salford M6 6PU, UK \\ ${ }^{\mathrm{d}}$ Greater Manchester Neurosciences Unit, Hope Hospital, Salford, UK \\ e Department of Clinical and Experimental Epilepsy, Institute of Neurology UCL, Queen Square, London WC1N 3BG, UK \\ ${ }^{\mathrm{f}}$ Epilepsy Society, Chesham Lane, Chalfont St Peter, Bucks SL9 ORJ, UK \\ ${ }^{\mathrm{g}}$ Max Planck Institute for Psycholinguistics, Wundtlaan 1, 6525 XD Nijmegen, The Netherlands
}

\section{A R T I C L E I N F O}

\section{Article history:}

Received 21 May 2011

Received in revised form

12 July 2011

Accepted 2 August 2011

\section{Keywords:}

Co-speech gesture

Communication

Non-verbal communication

Parkinson's Disease

Action

\begin{abstract}
A B S T R A C T
Introduction: Parkinson's disease (PD) can impact enormously on speech communication. One aspect of non-verbal behaviour closely tied to speech is co-speech gesture production. In healthy people, co-speech gestures can add significant meaning and emphasis to speech. There is, however, little research into how this important channel of communication is affected in PD.

Methods: The present study provides a systematic analysis of co-speech gestures which spontaneously accompany the description of actions in a group of PD patients $(N=23$, Hoehn and Yahr Stage III or less) and age-matched healthy controls $(N=22)$. The analysis considers different co-speech gesture types, using established classification schemes from the field of gesture research. The analysis focuses on the rate of these gestures as well as on their qualitative nature. In doing so, the analysis attempts to overcome several methodological shortcomings of research in this area.

Results: Contrary to expectation, gesture rate was not significantly affected in our patient group, with relatively mild PD. This indicates that co-speech gestures could compensate for speech problems. However, while gesture rate seems unaffected, the qualitative precision of gestures representing actions was significantly reduced.

Conclusions: This study demonstrates the feasibility of carrying out fine-grained, detailed analyses of gestures in PD and offers insights into an as yet neglected facet of communication in patients with PD. Based on the present findings, an important next step is the closer investigation of the qualitative changes in gesture (including different communicative situations) and an analysis of the heterogeneity in co-speech gesture production in PD.
\end{abstract}

(c) 2011 Elsevier Ltd. All rights reserved.

\section{Introduction}

Parkinson's Disease (PD) is a progressive neurodegenerative disorder in which death of dopaminergic cells in the substantia nigra pars compacta results in motor impairments. Normal motor functioning is not only crucial for navigating through the environment; it also underpins communicative acts such as speech and facial expression.

\footnotetext{
* Corresponding author. Tel.: +44 161275 7333; fax: +44 1612752588.

E-mail address: Ellen.Poliakoff@manchester.ac.uk (E. Poliakoff).
}

A number of verbal communicative deficits arise in PD due to motor difficulties; abnormal articulation, prosody [1], and difficulties modulating voice pitch and loudness [2]. PD patients also exhibit non-verbal communicative deficits, specifically difficulty generating [3] and recognising [4] facial expressions. However, the umbrella term of 'communication' involves more than speech and facial expressions; importantly it also encompasses co-speech gestures [5].

Co-speech gestures involve movements of the hands and arms, which healthy speakers produce spontaneously and frequently while talking [6]. Iconic gestures, one type of co-speech gesture, represent semantic information relating to concrete concepts, while metaphoric gestures occur with reference to abstract concepts [6]. Deictic gestures are gestures with a pointing function, 
including the use of the extended index finger or the whole hand to indicate a referent $[5,6]$. Other gestures play important interactive [7] as well as pragmatic [5] functions in dialogue. Beats, one form of pragmatic gesture, are simple biphasic flicks of the hand providing emphasis to the verbal message and are closely tied to the rhythmical pattern of speech [6]. Overall, co-speech gestures add important information above and beyond that communicated in speech [8]. However, despite the fact that reduced bodily gestures is a recognised feature of $\mathrm{PD}$ [1], the domain of co-speech gestures remains largely unexplored in $\mathrm{PD}$.

A small number of studies have investigated gesture production more generally in $\mathrm{PD}$, reporting a reduction in gesture use $[3,9]$. On the one hand, this is not surprising given patients' difficulties in producing fine, simultaneous and sequential movement [10]. Moreover, reduced gestures may be expected in parallel with reduced speech in PD, considering that gesture and speech form a single system [6]. On the other hand, this close connection also means that gestures could compensate for difficulties with spoken language [11]. This process can be observed in healthy speakers, for example in the context of verbal ambiguity [12] and lexical access [13].

One limitation of previous studies into gestures in PD is that rather than considering co-speech gestures, they have only considered those that occur in the absence of speech. For example, examining pantomime movements (e.g., brushing teeth) or imitating hand gestures [14]. Indeed, Ideomotor apraxia has been observed in a proportion of PD patients (27\% [15]). To date, however, only one study [3] has directly investigated the issue of co-speech gestures in PD. Their principle aim was to investigate which non-verbal cues resulted in PD patients being perceived as more 'hostile', 'anxious', 'morose', and less 'intelligent' than cardiac controls [16]. Consequently, they analysed the silent videotapes from this earlier study and concluded that 'gestures which commonly accompany speech' (p.182 [3],) are markedly reduced in PD patients compared to age-matched cardiac controls. However, their conclusions are limited by several factors; firstly, their analysis of silent videotapes is problematic. This is because these gestures are designed to go with speech, making reliable interpretation of people's idiosyncratic gestures [6] difficult in the absence of speech. Secondly, their categorisation of gestures included deictic gestures 'and others', namely 'all those associated with speech' (p. 180 [3],) - exactly which kind of gestures this comprised is left unspecified. Thirdly, their number of participants was very small $(N=4)$.

Therefore, the primary aims of this research were, firstly, to determine whether Parkinsonian patients gesture significantly less than age-matched controls and, secondly, to determine if any gesture type is particularly affected. Video footage was analysed of 23 patients and 22 healthy controls, describing two tasks involving everyday actions; pressing a button and turning a door handle. The co-speech gestures they produced during these descriptions were coded and analysed to answer the above research questions.

\section{Methods}

\subsection{Participants}

23 Parkinsonian patients and 22 healthy controls (Table 1 ) took part in this study, which was approved by the local research ethics committee. Participants consented to being video-recorded before the testing session began. All participants were screened for dementia at the start of the session using the Mini Mental State Examination [17] and scored in the normal range ( $>25 / 30)$. After the testing phase, but prior to video-recording, all participants completed a battery of questionnaires and standardised tests. As can be seen in Table 1, the groups did not significantly differ in age, intelligence quotient (IQ; estimated using the National Adult Reading Test [18] and the Mill Hill vocabulary test [19]), years of education, or on the Geriatric Depression Scale [20]

Patients were recruited at Hoehn and Yahr [21] stage III or less to enable them to perform the reaction time tasks. They scored a mean of $20.10(S D=6.60)$ on the motor subsection of the Unified Parkinson's Disease Rating Scale [22], with disease duration ranging from less than 1 up to 16 years. They completed the session "on" their normal medication, at a time of day they chose to maximise the chance of a stable on period, apart from one patient who was not yet taking medication. Twenty patients were taking L-dopa of which sixteen patients were also taking dopamine agonists. In addition, twelve patients were taking catechol-o-methyltransferase, three were taking anticholinergics and three patients were taking monoamine oxidase inhibitors. 18 of the patients exhibited tremor and 10 reported experiencing dyskinesias.

\subsection{Procedure}

The video data for this investigation was collected at the end of a session from a previously reported experimental study [23]. Each participant sat at a $45^{\circ}$ angle to the video-camera providing a full view of the participant's hands, arms, head and torso. They were asked to ignore the video-camera and to describe to the experimenter what they had done in the experimental session, imagining that the experimenter had been absent during the testing phase and therefore was unaware of what they had done. Briefly, in the first task, participants viewed a video clip of a blue square, or of a finger, moving up or down and were required to respond by pressing or releasing a button (Fig. 1A) when an "X" appeared onscreen. The second task involved pressing a left or right button (Fig. 1A) depending on whether an onscreen shape (a simple bar or door handle) was squared or rounded. As part of this task, participants estimated the time of real and imagined movements towards a metal door handle, mounted on a piece of wooden board and within reaching distance of the participant (Fig. 1B). After participants had described these tasks, the experimenter asked them to describe their favourite and least favourite aspect of the testing phase.

\subsection{Analysis}

The speech of each participant was transcribed verbatim from the videorecordings. The number of words (including word fragments and repeated words) was used to calculate their gesture rate per 100 words.

Gestural analysis included all arm, hand, finger and head movements which accompanied speech. Using the computer software ELAN (version 3.6.0), gestura movements were first identified from the video-recordings by a single coder. In most cases, the hand(s) returned to rest position after each individual gesture. When multiple gestures were produced in succession without the hand(s) returning to rest position, each stroke phase [6] was counted as one gesture. Gestures with direct verbal translation, namely emblematic gestures (e.g., 'thumbs up' gesture), as well as self-adapters (e.g., touching the hair or face [24],) were excluded as they bear a crucially different relation to speech compared to other co-speech hand movements. Each individual gesture was then classified (see Introduction) as being iconic metaphoric, deictic, or pragmatic/interactive (combining what some authors have defined as beats, interactive and pragmatic gestures). In total, 1878 gestures were identified. Finally, the number of gestures per 100 words was calculated for each

Table 1


Scale, MHVS) and depression (Geriatric Depression Scale).

\begin{tabular}{|c|c|c|c|c|c|c|c|c|}
\hline \multirow[t]{2}{*}{ Group } & \multirow[t]{2}{*}{ Gender } & \multirow[t]{2}{*}{ Age } & \multirow{2}{*}{$\begin{array}{l}\text { Years in } \\
\text { education }\end{array}$} & \multicolumn{2}{|c|}{ Digit span } & \multicolumn{2}{|l|}{ Intelligence } & \multirow[t]{2}{*}{ Depression } \\
\hline & & & & Forward & Backward & NART & MHVS & \\
\hline Controls $(n=22)$ & $\begin{array}{l}9 \text { male } \\
13 \text { female }\end{array}$ & $65.0(9.0)$ & $13(3)$ & $6.7(2.1)$ & $5.1(1.2)$ & $117.4(7.7)$ & $23.5(5.0)$ & $2.4(3.2)$ \\
\hline $\mathrm{PD}(n=23)$ & $\begin{array}{l}9 \text { male } \\
14 \text { female }\end{array}$ & $62.0(8.0)$ & $12(3)$ & $7.2(1.7)$ & $4.8(1.7)$ & $115.9(7.1)$ & $21.3(4.4)$ & $3.8(2.5)$ \\
\hline$t$-test & $N A$ & $\begin{array}{l}t=1.06 \\
p=.30\end{array}$ & $\begin{array}{l}t=.79 \\
p=.43\end{array}$ & $\begin{array}{l}t=.112 \\
p=.911\end{array}$ & $\begin{array}{l}t=.102 \\
p=.920\end{array}$ & $\begin{array}{l}t=.350 \\
p=.72\end{array}$ & $\begin{array}{l}t=1.36 \\
p=.18\end{array}$ & $\begin{array}{l}t=-1.47 \\
p=.15\end{array}$ \\
\hline
\end{tabular}




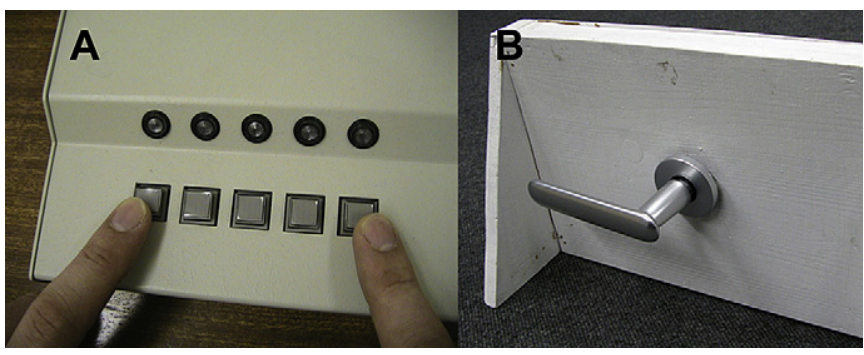

Fig. 1. The Button Box (panel A) and the door handle (panel B) used in the experimental tasks.

participant [(total number of gestures/total number of words)*100], thus controlling for differences in the length and rate of verbal utterances.

In order to establish reliability, $34 \%$ of the data $(N=629)$ was coded independently by a second judge, blind to the hypotheses of this study. Strong agreement was obtained for identifying (91.5\%, Cohen's Kappa $K=.83$ ) and categorising $(75.0 \%$, Cohen's Kappa $K=.69$ ) the gestures.

Interestingly, during coding, a qualitative difference became apparent in the data. Specifically, control participants seemed to exhibit more precision in their iconic gestural depictions of the actions 'door handle opening' or 'button pressing' than PD patients. To quantify this, the precision of all iconic gestures coupled with the description of these two actions was coded (100\% inter-rater reliability was obtained). A gesture for 'opening the door' was defined as precise if it included both a clenched hand, so that the fingers and thumb make an open or closed circle, and a downward motion. For 'pressing the button', the co-speech gesture was coded as precise if it included both the extension of a single finger (usually forefinger) and downward movement.

For the statistical analyses, parametric and non-parametric tests were used depending on normality of the data (Shapiro Wilk tests) and an alpha level of .05 was used throughout.

\section{Results}

To assess whether PD patients exhibited reduced co-speech gesture production compared to controls, we compared gestures per 100 words between groups. Although the PD patients $(M=8.97$, $S D=6.22$ ) produced numerically fewer gestures per 100 words than controls $(M=9.54, S D=7.00)$, this difference was not significant, $t(43)=.28, p=.77$, two-tailed. Nor did overall gesture rate correlate with motor UPDRS score, $r=-.054, p=.806$.

To investigate whether production of the different types of cospeech gestures differed from controls, independent $t$-tests and Mann-Whitney $U$ tests were used to compare the percentage of each gesture type between the groups (all two-tailed). None of the comparisons were significant (see Table 2). Note that three participants with PD produced no gestures and were excluded from this analysis as their data provided no information about proportional use of different gesture types. These 3 patients all exhibited tremor (action tremors of severity $1-3$ ) and $2 / 3$ experienced dyskinesias (moderately disabling for $25 \%-50 \%$ of the day).

To investigate whether the precision of gestures differed, the frequency of participants who exhibited one or more precise iconic gesture versus those who produced less precise iconic gestures was compared between groups (see Method section for definition of gesture precision). Individuals who did not gesture during the

Table 2

Measures of central tendency and dispersion [Mean (SD) and Median (Range) respectively], as well as test statistics, for the percentages of different gesture types produced by the two groups.

\begin{tabular}{lllll}
\hline Group & Iconic & Metaphoric & Deictic & Pragmatic \\
\hline Controls $(n=22)$ & $37.52(26.25)$ & $.00(14.75)$ & $15.25(92.86)$ & $37.36(31.90)$ \\
PD $(n=20)$ & $42.38(18.71)$ & $.00(17.24)$ & $22.65(50.79)$ & $31.74(22.37)$ \\
Test statistics & $t=.69$ & $U=208.00$ & $U=247.50$ & $t=.66$ \\
& $p=.50$ & $p=.69$ & $p=.49$ & $p=.52$ \\
\hline
\end{tabular}

Table 3

Frequencies of controls and PD patients who exhibited one or more precise iconic gesture in contrast to no precise gestures.

\begin{tabular}{lll}
\hline & $\begin{array}{l}\text { One or more } \\
\text { precise gesture }\end{array}$ & $\begin{array}{l}\text { No precise } \\
\text { gesture }\end{array}$ \\
\hline Control & 17 & 3 \\
PD & 7 & 8 \\
\hline
\end{tabular}

descriptions of these actions were excluded from this analysis ( $N=5$ in the PD group, $N=2$ in the control group) since their gestures could not be categorised as precise or not. A Chi-square test revealed a significant association, with controls typically producing precise gestural depictions whereas PD patients showed a tendency to using more imprecise gestures $\left(X^{2}(1)=5.84, p=.016\right.$, two-tailed; see Table 3$)$. It was found that the PD patients who produced one or more precise gesture exhibited a significantly higher overall gesture rate $(M=15.25, S D=3.30)$ than those who made less precise gestures $(M=8.19, S D=4.87 ; Z=2.20, p=.028)$. There was also a numerical trend for a lower motor UPDRS score for the precise group $(M=19.00, S D=5.94)$ compared to the less precise group $(M=23.37, S D=6.89)$. There was also a nonsignificant trend for the precise group to exhibit less severely affected speech within the UPDRS $(M=.71, S D=.76)$ than the less precise group $(M=1.25, S D=.89)$.

\section{Discussion}

Contrary to past research, the present study found no significant difference in the overall rate of co-speech gestures in this group of Parkinson's patients and age-matched controls, or in the proportions of individual gesture types. This suggests that there is not a simple reduction in gestural communication in PD. The fact that PD patients gestured statistically at the same rate as controls hints at the possibility that co-speech gestures could compensate for speech deficits in PD [11], since effects on speech emerge earlier than gesture [25]. However, the interaction of gesture and speech in the representation of semantic information [12] needs to be explored in PD to allow for more definite conclusions.

Further underlining the importance of such a semantic analysis is our second finding; despite no difference in gesture rate, PD patients showed a reduction in the semantic precision of iconic gestures associated with the depiction of particular actions (pressing a button, opening a door). This fits with previous observations of less well-formed gestures [10] and kinematic deficits [26] when PD patients pantomime actions. One possible explanation for this effect is an impairment of the mental representation underlying the gestures. Indeed, PD patients have particular difficulties with the cognitive processing of action information (as compared to objects, for example [27]). With respect to gestures, PD patients' difficulty relating to actions might be directly associated with the cognitive generation of the underlying imagistic aspect of the motor component forming part of the gesturally depicted action. Further research is required investigating gestural communication about a broader range of information (e.g. including more abstract topics) to determine whether gestures are generally less precise, and thus less informative, in PD or if this finding is specific to mental representations involving a manual motor component.

Although the current study was not designed to assess the effect of disease severity and heterogeneity on gesture production in PD, and included patients only at mild to moderate disease stages tested "on" medication, the results suggest a complex picture which warrants further investigation. On the one hand, our results suggest some association between disease progression and the rate and precision of co-speech gesture production; the group who 
produced less precise gestures produced fewer gestures overall compared to the precise group and tended to have a higher level of motor and speech symptoms. On the other hand, overall gesture rate did not correlate with the degree of motor disability. Furthermore, while the three patients who did not produce any gestures all exhibited tremor and dyskinesias, these symptoms were also apparent (and of equivalent severity) in the precise gesture group. This suggests that gesture production must also be influenced by non-motor factors, such as embarrassment (cf [28]) which may cause avoidance of gestures. Further, co-speech gesture production may also be influenced by general cognitive problems seen in PD, such as impairments in spatial working memory or divided attention [29]. One possibility is that coordinating speaking and gesturing is more difficult than speaking alone. However, this is unlikely as co-speech gestures have been found to 'lighten' a speaker's cognitive load [30].

A possible criticism of the current study is that dyskinetic symptoms may have been mistakenly identified as gestures, increasing the apparent gesture rate in the PD group. We believe this is unlikely as this would produce a rise in the number of pragmatic/interactive gestures, and especially beats (i.e., simple, non-referential and often biphasic movements of the hands) and not other types (i.e., those that convey semantic information). A further limitation is that participants were describing the experimental procedure in which they took part to a recipient whom they knew was already familiar with it (the experimenter). While future research should aim to overcome this limitation, this communicative situation was the same for both participant groups and is therefore unlikely to account for our results.

The present findings demonstrate the applicability of a more fine-grained gestural analysis to the PD population, showing that although the PD patients did not show a significant reduction in gestures rate, the qualitative nature of the gestural depictions seemed to be affected. The findings encourage further enquiry, in particular a systematic analysis of the semantic interplay of gesture and speech in PD and an investigation of the relationship between motor symptoms, speech production and gesture production in a larger sample of patients. Another interesting future avenue would be the comparison of gestures (and communication) on and off medication. Further investigation of this modality of communication is important in advancing our understanding of PD, but also in relation to the question of how people with PD, and those that engage with them, might improve communication.

\section{Acknowledgements}

This work was supported by Parkinson's UK [grant number 8043]. We thank the patients and controls for participating, Samantha Rowbotham for performing the inter-rater reliability and the Wellcome Trust Clinical Research Facility Manchester for providing testing facilities.

\section{References}

[1] Scott S, Caird F, Williams BO. Communication in Parkinson's Disease. London: Croom Helm; 1985.
[2] Ramig LO, Dromey C. Aerodynamic mechanisms underlying treatment-related changes in vocal intensity in patients with Parkinson Disease. J Speech Hear Res 1996;39:798-807.

[3] Pitcairn TK, Clemie S, Gray JM, Pentland B. Non-verbal cues in the selfpresentation of Parkinsonian patients. Brit J Clin Psychol 1990;29:177-84.

[4] Sprengelmeyer R, Young AW, Mahn K, Schroeder U, Woitalla D, Büttner T, et al. Facial expression recognition in people with medicated and unmedicated Parkinson's disease. Neuropsychologia 2003;41:1047-57.

[5] Kendon A. Gesture: visible action as utterance. Cambridge: Cambridge University Press; 2004.

[6] McNeill D. Hand and mind: what gestures reveal about thought. Chicago: University of Chicago Press; 1992.

[7] Bavelas J, Chovil N, Lawrie D, Wade A. Interactive gestures. Discourse Process 1992;15:469-89.

[8] Holler J, Shovelton H, Beattie G. Do iconic hand gestures really contribute to the communication of semantic information in a face-to-face context? J Nonverbal Behav 2009;33:73-88.

[9] Duncan S. Gestural imagery and cohesion in normal and impaired discourse. In: Wachsmuth I, Lenzen M, Knoblich G, editors. Embodied communication in humans and machines. Oxford: Oxford University Press; 2008. p. 305-28.

[10] Sharpe HM, Cermak SA, Sax DS. Motor planning in Parkinson patients. Neuropsychologia 1983;21:455-62.

[11] Miller N, Noble E, Jones D, Burn D. Life with communication changes in Parkinson's disease. Age Ageing 2006;35:235-9.

[12] Holler J, Beattie G. Pragmatic aspects of representational gestures: do speakers use them to clarify verbal ambiguity for the listener? Gesture 2003;3:127-54.

[13] Beattie G, Coughlan J. An experimental investigation of the role of iconic gestures in lexical access using the tip-of-the tongue phenomenon. Brit J Psychol 1999;90:35-56.

[14] Zadikoff C, Lang AE. Apraxia in movement disorders. Brain 2005;128: 1480-97.

[15] Leiguarda L, Pramstaller PP, Merello M, Starckstein S, Lees AJ, Marsden CD. Apraxia in Parkinson's disease, progressive supranuclear palsy, multiple systems atrophy and neuroleptic-induced parkinsonism. Brain 1997;120: 75-90.

[16] Pentland B, Pitcairn TK, Gray JM, Riddle WJR. The effects of reduced expression in Parkinson's disease on impression formation by health professionals. Clin Rehabil 1987;1:307-13.

[17] Folstein MF, Folstein SE, McHugh PR. “Mini-Mental State” A practical method for grading the cognitive state of patients for the clinician. J Psychiatr Res 1975;12:189-98.

[18] Nelson HE. National Adult Reading Test (NART). 3rd ed. London: NFER-Nelson; 1991.

[19] Raven JC. Guide to using the Mill Hill Vocabulary Scale with the Progressive Matrices Scales. London: HK Lewis And Co. Ltd; 1958.

[20] Sheikh JI, Yesavage JA. Geriatric Depression Scale (GDS): recent evidence and development of a shorter version. Clin Gerontol 1986;5:165-73.

[21] Hoehn M, Yahr MD. Parkinsonism: onset, progression and mortality. Neurology 1967; 17:427-42.

[22] Fahn S, Elton RG. Members of the UPDRS development committee: Unified Parkinson's Disease Rating Scale. In: Fahn S, Marsden CD, Calne DB, Goldstein M, editors. Recent developments in Parkinson's disease. Florham Park (NJ): Macmillan Healthcare Information; 1987. p. 153-63.

[23] Poliakoff E, Galpin A, Dick JP, Moore AP, Tipper SP. The effect of viewing graspable objects and actions in Parkinson's Disease. NeuroReport 2007;28: 483-7.

[24] Ekman P, Friesen WV. The repertoire of nonverbal behavior: categories, origins, usage, and coding. Semiotica 1969;1:49-98.

[25] McNamara P, Durso R. Pragmatic communication skills in patients with Parkinson's disease. Brain Lang 2003;84:414-23.

[26] Leiguarda L, Mercello M, Balej J, Starkstein S, Nogues M, Marsden CD. Disruption of spatial organization and interjoint coordination in Parkinson's disease, progressive supranuclear palsy and multiple system atrophy. Mov Disord 2000;15:627-40.

[27] Signorini M, Volpato C. Action fluency in Parkinson's Disease: a follow-up study. Mov Disord 2006;21:467-72.

[28] Traub RE, Gerbin M, Mullaney MM, Louis ED. Development of an essential tremor embarrassment assessment. Parkinsonism Relat Disord 2010;16:661.

[29] Kemps E, Szmalec A, Vandierendonck A, Crevits L. Visuo-spatial processing in Parkinson's disease: evidence for diminished visuo-spatial sketch pad and central executive resources. Parkinsonism Relat Disord 2005;11:181-6.

[30] Goldin-Meadow S, Nusbaum H, Kelly S, Wagner S. Explaining math: gesturing lightens the load. Psychol Sci 2001;12:516-22. 